\title{
Erratum to: A Comparative Study of Different Necking Criteria for Numerical and Experimental Prediction of FLCs
}

Cunsheng Zhang, Lionel Leotoing, Guoqun Zhao, Dominique Guines, and Eric Ragneau

\section{Erratum to: J. of Materi Eng and Perform}

DOI 10.1007/s11665-010-9729-7

The following acknowledgement was missing from the article as published:

\section{Acknowledgment}

This work was financially supported by Encouragement Foundation for Young Scholars of Shandong Province (BS2010ZZ004).

The online version of the original article can be found under doi:10.1007/s11665-010-9729-7.

Cunsheng Zhang and Guoqun Zhao, Key Laboratory for LiquidSolid Structural Evolution and Processing of Materials, Ministry of Education, Shandong University, 73 Jingshi Road, Jinan 250061, Shandong Province, People's Republic of China; and Lionel Leotoing, Dominique Guines, and Eric Ragneau, Université Européenne de Bretagne, INSA-LGCGM-EA 3913, 20, Avenue des Buttes de Coësmes, 35043 Rennes Cédex, France. Contact e-mails: zhangcs@ sdu.edu.cn and zhaogq@sdu.edu.cn. 